\section{CASE II.}

On June 9th, 1924, I was called in consultation by Dr. E. $\mathbf{R}$. Weir of Dunoon to see an unmarried woman, aged 68, who had been suffering from abdominal pain and vomiting for forty-eight hours. The signs of acute intestinal obstruction were complete, but the romit was not faecal.

Operation was immediately undertaken. On inserting a hand into the abdomen I came at once upon a mass in the ileum four or five inches proximal to the ileo-caecal valve. Through a small incision in the bowel 1 removed a gall stone. Recovery was uneventful.

was uneventful.

This calculus was roughly a truncated cone. The greatest length equalled the greatest breadth-namely, one and one-fifth inches. The dry weight was 180 grains.

\section{CASE III.}

A detailed account of this case has already been published in another commexion. ${ }^{2}$ The operation took place on January 10th 1917. A married woman, aged 52, had had a long experience of so-called gastric disease before iniestinal obstruction manifested itself. The stone which I removed from the ileum was ovoid in shape, the greatest circumference being $5 \frac{1}{2}$ inches, and the weight was 636 grains. Recovery was uninterrupted.

1 saw this patient recently, more than eight years after operation, when she reported well, and stated that she had had no recurrence of discomfort.

It is noteworthy how seldom these patients have earlier symptoms pointing directly to the gall bladder; that there should sometimes be complete absence of any morbid signs before the onset of obstruction is equally striking.

It is scarcely ever possible to diagnose the cause of the obstruction before operation, but during the operation the mass in the intestine is easily discovered by the exploring hand. The bowel, tightly gripping the impacted stone, shows signs of bruising by the irregular surface of the calculus, and the direction of the incision into the intestine must be determilied by the injury already sustained by the intestinal coats.

On no occasion have I attempted, as advised by several authorities, to move the stone along the interior of the bowel before cutting down upon it, as I am of opinion that the less the damaged intestine is handled the better.

It is now generally believed that gall bladier and small intestine adhere together on account of an inflammatory process, and that the large calculi pass directly into the intestine through a stoma set up by a process of ulceration.

There can be little doubt that these patients, when they have recovered from the operation for intestinal obstruction, derive considerable benefit from the cholecystenterostomy established by this curious happening. By this means they become possessed of two outlets from the gall bladder, and a period is thereby put to this organ acting as a factory of gall stones.

REFERFNes.

August, 1917.

TUBERCULOSIS IMMUNIZATION. BY

NATHAN RAW, C.M.G., M.D., M.R.C.P.Lonמ., LORD CHANCELLOR'S VISITOR IN LUNACY.

Iv the British Medical Journal of July 19th, 1924 (p. 102), ] described a series of animal experiments in which it was conclusively shown that it is possible to give active immunization to animals against a virulent dose of tubercle bacilli.

A further series of experiments has just been concluded at the Lister Institute with the same encouraging results, and I am hopeful that in the near future the work will be extended to young children, with a view to protecting thase who are in daily contact with cases of open tuberculosis.

Animal Experiments.

-A large series of rabbits and guinea-pigs were used. Half of the animals were given protective doses of vaccine prepared from attenuated killed human bacilli, which had been continuously subcultured by me for sixteen years, and were quite non-pathogenic and non-tuberculogenic. Two protective doses were given-one on July 25th, 1925, and the other on August 1st. The whole series of animals, including the controls, were then given $0.1 \mathrm{mg}$. of virulent bovine bacilli by Dr. Schutze.

One of the rabbits and two guinea-pigs died in three weeks from septicaemia, and the whole of the controls died within three months of acute and generalized tuberculosis. The protected animals gained weight and appeared quite healthy when they were killed on February 24th, 1926. A very careful post-mortem examination of the rabbits was made by Dr. Schutze and myself. The animals were fat and in excellent condition. At the site of inoculation (in each case in the thigh) was an enlarged gland or glands, but not extending into the inguinal glands. There was no trace of tubercle in any of the organs. Smears from the glands showed tubercle bacilli in large numbei's, and these will be injected into guinea-pigs with a view to testing their virulence. It is evident that a local infection was produced at the site of inoculation, but the animals were protected against a progressive tuberculosis.

As my previous experiments have also shown, it is only possible to protect animals by using bacilli of human origin, against an infecting dose of bovine origin, and vice versa.

\section{Conclusions.}

The problem of tuberculosis resolves itself into the question of immunity. Contrary to what is observed in other infectious diseases, no immunity is conferred on the human by a previous attack of tuberculosis, caused by the same type of bacilli. Pulmonary tuberculosis, which is nearly always caused by bacilli of the human type, does not appear to give immunity in after-life, and patients whose disease has been arrested are liable after many years to another acute attack of active tuberculosis. Clinical experience, however, goes to show that children and young adults who have received a mild infection of bovine bacilli in milk, causing enlarged glands in the neck, bone and joint lesions, and other forms of localized tuberculosis, are practically immune to pulmonary tuberculosis, and it was this observation that led me to use bovine bacilli to immunize animals and man against the more serious disease of the lungs.

It seems to be probable that children may be protected against infection in the household, but some years must elapse before this can be finally established. All children over the age of 1 year who are living in the same house with a tuberculous member of the family ought to be protected if possible against infection, and it is in the early years of life that such infection usually occurs.

From a long observation of tuberculosis I am convinced that if some active immunity can with complete safety be given to those children who are directly expased to infection at home, a long step will have been taken in the direction of prevention, which, after all, is the key to the problem.

The protection of the community by some safe scientific method of active immunization is the only effective way by which this preventable scourge can be eradicated.

\section{FRACTURE OF THE SKULL AND EXTRADURAL HAEMORRHAGE WITH SYMPTOMS OF HYPOTENSION.}

\section{BY} SYDNEY SMITH; M.D., D.P.H., PRINCJPAL MEDICO-LETAL EXPERT, EGYPTIAN GOVERNMEAT SERVICE.

THE case recorded below is of much interest, since, although there was considerable extradural haemorrliage, there was a complete absence of pressure signs. The case suggests that a haemorrhage within the skull may act not only by its own local pressure but also by its general effect on the pressure of the cerebro-spinal fluid of the brain.

A man, aged 26, was knocked down. by a tram on November 27 th and was taken to hospital in a comatose condition.

On admission the pulse was 100 and irregular, the temperature $38^{\circ} \mathrm{C}$., and the respirations shallow. There was escape of bloor from the nose and the right ear. The pupils were unequal and 